Relevance of the irrelevant: using taskirrelevant emotional stimuli to test the loadhypothesis through ERP's.

Handledare: Stefan Wiens

VETENSKAPLIG UNDERSÖKNING, PSYKOLOGI III, 30 HP, HT 2009

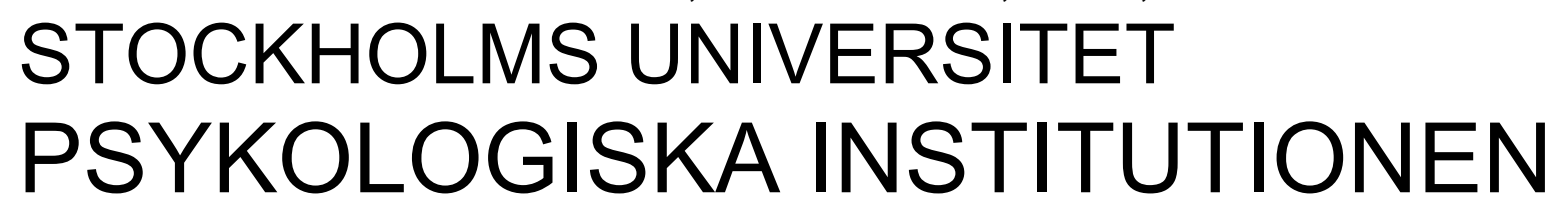




\title{
RELEVANCE OF THE IRRELEVANT: USING TASK-IRRELEVANT EMOTIONAL STIMULI TO TEST THE LOAD-HYPOTHESIS THROUGH ERP'S.
}

\author{
Per Andersson
}

\begin{abstract}
The role of attention and perceptual resources were studied in a one-back task and a letter-search task, both using the same stimuli. In the letter task, pictures were used as task-irrelevant and distracting emotional stimuli. The emotional processing of the pictures was measured through the Late Positive Potential (LPP), an event-related potential (ERP) recorded with EEG. LPP activity was significantly greater to emotional than neutral stimuli during the one-back task; this shows that emotional stimuli were processed during an easy task (low load). However, LPP activity dropped for all stimuli during the difficult perceptual task (high load). Selective processes of attention are discussed, in relation to Load Theory and the ability to ignore task-irrelevant, but emotionally significant, stimuli.
\end{abstract}

Perception is more than meets the eye. Though the physiological mechanisms of the eye have long been mapped, this has not given us a complete understanding of perception. To understand perception, it is not enough to map the eye, as this is only the producer of visual output. The process of perception itself should rather be investigated inside the brain, where this output is processed further. Consequently, this has been the subject of much research.

In an environment rich in stimuli, only some stimuli capture our attention. Attention is here seen as directing more processing resources to some particular stimuli over other stimuli, sometimes to the point of completely ignoring other stimuli. That attention affects perception becomes evident in studies of inattentional blindness, where participants may become perceptually blind to major changes in their field of vision (such as a gorilla walking past) if attention is focused on other aspects of the same set of visual stimuli (Koivisto \& Revonsuo, 2008; White \& Davies, 2008). Even though participants would generally perceive such an unexpected stimulus, as it is presented in clear view, and stands out, it seems that the tasks that participants are given to guide attention prevents the perception of this unexpected stimulus.

At some level, selection of available stimuli occurs. Certain stimuli seem to be prioritised as opposed to others, and only selected stimuli are reacted on. As the selective process shows regularities in stimulus selection, it cannot be at random, but instead using some system of priorities. Studies show that it is not exclusively task-related, as certain types of stimuli appear to be prioritised regardless of task. For instance, in visual search tasks, participants are generally quicker at finding emotionally significant stimuli, in the form of fearrelevant snakes and spiders (Öhman, Flykt, \& Esteves, 2001) or human faces (Devue, Laloyaux, Feyers, Theeuwes, \& Brédart, 2009) than more neutral forms of stimuli, even when the task is essentially the same. Processes of pattern 
recognition can be fully automatic, and genetically passed down. A study of fivemonth old human infants showed that they focused their attention longer on an image of a fear-relevant spider, as opposed to other, more neutral stimuli (Rakison \& Derringer, 2007). Emotional stimuli may also interfere with a current task and impair performance, even when participants are asked to ignore them (Norberg, Wiens \& Peira, 2009). While current task focus may be shifted quickly on a conscious level, as participants switch between tasks according to instructions given to them, automatic processes, such as certain emotional reactions, are not as easily altered (conscious should here be understood as having explicit access to a process, also see discussion). Some basic fear responses are not quickly modified, and may be the result of an evolutionary adaptation process to a former natural environment, as becomes evident in the case of spider phobia (Buss, 2009). Reactive emotions and conscious task focus both affect the selective process. What remains unclear is how this selection operates. For example: it is not yet fully understood how priorities are handled between conscious and unconscious processes.

According to the theory of Early Selection (Lavie, 1995, for a historical review), there exists a bottle-neck in systems of perception. Only so much perceptual stimuli can be processed at once, and so irrelevant stimuli are filtered away. The filtering of stimuli can be dependent on conscious goals (task focus) as well automatic processes (i.e. emotions), but takes place already at an early level. Selection at an early level would mean that stimuli are processed by simple physical qualities such as colour and shape, and selected according to these (Lavie, 1995). Should this be the case, it would mean that unattended stimuli would not be processed beyond its most basic features. It would also mean that we would not have conscious access to (would not be able to become aware of) semantic properties, such as object identity and meaning, of unattended stimuli (Conway, Cowan \& Bunting, 2001). We would then be limited in our reactions, in that we could only react to the stimulus that has made it through the filter processes.

The Early Selection theory has been disputed, however, and a theory of Late Selection has been suggested (Lavie, 1995, for a historical review). This theory dictates that selection occurs only after the processing of semantic properties, that the processing resources are unlimited, and that all stimuli remain available to react to (Lavie, 1995). However, as we cannot react to everything, a selection is made, but only after we have obtained information about the identity of all available stimuli. This would mean that even unattended stimuli may give rise to later processing, and that distracters are available to react to, but not necessarily prioritised for reaction. Also, it could possibly allow for direct conscious access to the filtering process.

The debate over Early or Late selection is still ongoing (Lachter, Forster \& Ruthruff, 2004). Now, there is evidence for both alternatives. However, this need not be paradoxical, if one introduces a third theory, The Load Theory (Lavie \& Tsal, 1994). If the thesis was Early Selection, and the anti-thesis Late Selection, then Load Theory could prove a viable synthesis of the two. Load Theory, proposed by Lavie \& Tsal (1994) dictates that under situations of low perceptual load, late selection occurs, and under situations of high load, early 
selection occurs (Lavie, 2005). In Load Theory, visual perception is presented as an ongoing automatic process, which continues without the need for conscious instruction, but can be directed. There are not endless perceptual resources available, and so resources are directed depending on different factors, including current task. If a task does not use all existing resources, meaning the perceptual load is low, some resources will spill over to taskirrelevant stimuli automatically. However if a task is perceptually demanding, stimuli that would be otherwise noticed might not be processed, leading to inattentional blindness to this stimuli, or rather, a load-induced blindness (Macdonald \& Lavie, 2008).

Perceptual load is varied by the resources needed to complete a visual task, yet it is not reducible to task difficulty, even if it can reflect one aspect of difficulty. Where difficulty may be manipulated by data limits, through making targets more difficult to spot in a number of ways, perceptual load is only varied under such circumstances in which more attentional resources can be allocated to better perceive the target (Lavie \& Fockert, 2003). One might, for instance, be trying to determine the identity of an infinitely small letter, however more perceptual resources cannot aid in this. If one had more perceptual resources, at any given time, then one would be able to process more of the present stimuli, but it would not necessarily improve the perception of the single stimulus.

In studies of perceptual load, load is manipulated mainly through increasing or decreasing the number of potential targets, here called standards, in a visual search task (Bahrami, Carmel, Walsh, Rees \& Lavie, 2008). In such tasks, there is typically one target that is to be searched for, a number of standards to increase or decrease load, and the task irrelevant stimuli, the distracter. The distracter is irrelevant to the task, in the way that it will not aid in any way to solve the task, yet it remains distracting as it is processed as long as there are still perceptual resources left to process it. Simply instructing participants to ignore a stimulus is not enough, as perception is an automatic process. There needs to be a perceptual load, to use all available resources, to enable blindness to irrelevant stimuli (Lavie, 2005). Evidence in support of load effects includes brain-imaging studies. For instance, responses in V5 of the visual cortex to a moving distracter decreased with load (Rees, Frith \& Lavie, 1997) and functional imaging studies typically show that neural signatures of irrelevant stimuli are eliminated entirely at high perceptual load (Macdonald \& Lavie, 2008).

Reactions to task-irrelevant stimuli is a key to explore the fundamental nature of attention. Through understanding under what circumstances the irrelevant stimuli are still processed, and at what level, it is possible to draw conclusions as of at what level the perceptual selection occurs. Measuring the effects of task-irrelevant stimuli can be tricky. Response time has been frequently used for this purpose (Lavie, 2005). A longer response time is thought to signify greater distracter effects, but can also signify greater general difficulty. Also, even if the distracter effect was isolated from difficulty, an increase in response time alone would not tell us at what level the distracter interferes. It is possible to use the distinction between explicit and implicit memory to determine effects, 
but this requires participants to take some form of test after each occurrence of a distracter, in order to see if it was consciously perceived, if it yielded priming effects, or if it yielded no effects at all. There are also other methods, that can be time-consuming and tedious for the participant, and often comes with the cost of issues of subjectivity and fallibility of participant feedback. To use a straight-forward physical measure would greatly aid, then.

Tracking eye movements would at first glance seem a possible method to measure distracter perception. However, studies have pointed towards the existence of two separate mechanisms of attention: eye fixation and covert attention (Lachter et al., 2004). Where eye movement and fixation is directly observable, covert attention is not. Covert attention is the mechanism of directing attention to different parts of the visual field, without corresponding eye-movements (making it is possible to focus attention on stimuli at the corner of the eye). Also, eye movement alone tells us little about where the movement originated and later perceptual processing. Even though participants generally tend to move their eyes in the direction of attention, methods such as short exposure times may prevent this movement (Beck \& Lavie, 2005).

By using distracters with physical effects on participants, objective measurements can become a possibility, if it can be established that these physical effects occur only when the distracter is processed, and are accurately measurable. Then, by using distracters that cause emotional responses, in an environment that otherwise lack emotionally significant stimuli, their effect could become isolated. In order to test emotional responses to distracters, Norberg et al. (2009) showed task-irrelevant pictures of spiders, to spider fearful and nonfearful participants. The emotional responses were measured through EEG (Electroencephalography) potentials related to emotional processing. Their study showed that even at high load, fearful individuals still responded emotionally to the task-irrelevant stimuli. As these findings were either contrary to Load Theory, or evidence that there exist some stimuli that is so highly emotional that it cannot be ignored, even at high load, it called for further investigation.

\section{Event-related potentials in EEG}

Readers already familiar with ERP-methodology may choose to skip this part. With EEG (Electroencephalography), measurements of cortical activity in the brains of wake humans have been made since the 1930's (Luck, 2005). As processes taking place in the brain produce distinct response patterns of brain activation, it is possible to analyze what processes are ongoing in the brain, by pinpointing what regions that are active. Activity in non-intrusive EEG is measured by electrodes placed on the scalp level, capable of sensing positive and negative voltage changes. The potential difference in voltage between two selected electrodes can be displayed as a waveform. Usually, activity in a region is measured as the difference between the active electrodes at the region of interest, and one or several reference electrodes, placed were activity is generally stable. Theory dictates that activation of specific areas in the cortex are dependent on networks of pyramidal neurons that use electrochemical signalling. The signalling process creates postsynaptic potentials, turning postsynaptic neurons into dipoles, producing electric fields. Larger groups of 
pyramidal neurons activated together will produce changes in voltage measurable at scalp level.

Post-synaptic potentials are not the only source of electrical activity measurable by EEG, however. Spontaneous activity (EEG rhythms, sometimes used to assess mental state of awareness) is always occurring in the brain, and it differs somewhat between individuals. There may be background electrical activity related to equipment as well. Also, activity related to movement, such as eye blinks, affects measurements by creating artefacts. This activity might sometimes be of interest, but this was not the case here. Luckily, as computer programs were developed for use of after-recording treatment of data, it became possible to isolate smaller changes in voltages and amplitudes from this other noise. It was then possible to investigate potentials that change as an effect of events taking place outside the brain. For instance, it became possible to see how activity was affected by presenting certain visual stimuli. These small changes in voltages, related to outside events, are called event-related potentials (ERPs). The method of ERP recording is to single out the specific waveforms that are responses to a certain external event (more about this in the method section). Already at $100 \mathrm{~ms}$ after visual stimulus presentation, it is possible to discern a clear positive waveform, called P100, that changes in relation to presented stimulus (Luck, 2005). There are several other, later components. The Late Positive Potential (LPP), one of these later components, was the focus of this study.

\section{The Late Positive Potential}

The LPP is a recorded positivity at central-parietal, midline sites, during the 300 $500 \mathrm{~ms}$ following certain visual stimuli. Series of studies have shown that stimuli rated as emotional produce much larger positive potentials, for both positive and negative emotional stimuli as compared to neutral (De Cesearei \& Codispoti, 2006; Hajcak, Dunning \& Foti 2007; Pastor, Bradley, Löw, Versace, Moltó \& Lang, 2008; Schupp, Cuthbert, Bradley, Cacioppo, Ito \& Lang, 2000).

LPP is seen as a relatively automatic response to emotional stimuli, as it is reoccurring, pinpointed in time as following the stimuli presentation and occurs without the need for specific instructions related to the stimuli presented. Though there seems to be a cognitive component to the LPP, as the same set of stimuli may give rise to different LPP amplitudes, depending on where attention is focused. For instance, if participants focused on the less arousing aspects of a negative emotional stimuli, LPP amplitudes dropped significantly compared to when they were simply viewing the same stimuli (Hajcak et al., 2007). The LPP is then affected by attention, and should be considered a postperceptual process, linked to stimulus evaluation rather than selection. Attentional and motivational factors influence LPP amplitudes, and LPP is greater when stimuli are emotionally significant (de Cesarei \& Codispoti, 2006). Viewed as a topography, the LPP can typically be identified as an increased positivity, originating from zones at both left and right hemispheres at the parietal lobe, forming two "peaks" that converge over time. 
The present study

This study explored how LPP activity was affected by the emotional content of a task-irrelevant distracter, and differences in task. The hypothesis, building on load theory (Lavie \& Tsal, 1994), was that a low-load task would allow for LPP activity to emotional stimuli, while a high-load task would not. Two tasks were conceived for the experiment. The first task was a manipulation check to show that LPP activity was affected by the emotional content of the stimuli, when attention was directed at it. A viewing task was selected for this purpose, in the form of the one-back task.

The other task tested how LPP activity was affected when a perceptual-load task was introduced, using the same stimuli, but attempting at directing task attention away from the emotional stimuli. A letter-search task, similar to that of earlier studies of spider fearful individuals, was chosen (Norberg et al., 2009). However, the focus was placed on showing similar stimuli, in the form of highly arousing and negatively emotional images, yet other than spiders and snakes. It was predicted that LPP activity would be lower for the more perceptually demanding of the two tasks, and also lower for neutral as opposed to emotional images presented.

Method

\section{Participants}

Fourteen students of Psychology (7 males, 7 females) were recruited, from corridors and classrooms across the department of Psychology at the Stockholm University, to participate in the experiment. The age of the participants ranged from 18 to 29 years $(\mu 24.0, S D 3.90)$. As compensation for their participation, they were rewarded with either movie vouchers or class credit. Participants had not previously been exposed to the chosen stimuli, and signed an informed consent form. Those with visual impairments were allowed to correct their vision by means of spectacles and contact lenses.

\section{Stimuli}

The experiment was run on a 21-inch monitor at a resolution of $1024 \times 768$ and a refresh rate of $100 \mathrm{~Hz}$. The experimental presentation was programmed and executed using Presentation software (Neurobehavioral Systems, www.neurobs.com).

300 pictures were selected from the IAPS library (Lang, Bradley \& Cuthbert, 2008 ) for the stimuli condition. Of these, 150 were high in arousal and negative in valence and the remaining 150 low in arousal and neutral in valence. The selection was based on a sample of IAPS images used in previous studies, but with the elimination of all pictures using frames, in an effort to keep images the exact same size without filler frames. Also, as pictures of spiders and snakes had been shown to cause effects resistant to perceptual load in earlier studies, these were omitted. Of the 300 images used, a selection of 20 was used exclusively for training-trials. Three images (IAPS numbers $2799,3350,6360$ ) 
were resized as to eliminate their black frames, thereby effectively altering their content slightly, but these were only used for the training trials, and so no data was recorded for these.

\section{Procedure}

The experiment consisted of two tasks. In the viewing task, participants looked for a repetition of a picture and to press a key every time a repetition of an image (target) occurred. Pictures were only repeated in direct succession, so participants had to remember only the most recent picture, (one-back). The viewing task was conceived as a condition of no (or extremely low) perceptual load.

The letter task focused on the letters surrounding the picture and the objective of the task was to find the letter $\mathrm{N}$ (target). Participants had to press a key every time they saw the letter $\mathrm{N}$. Of the six letters that were shown, there were either five N's or none, in any presentation. Letters randomly changed at each trial, and positions not occupied by an N-letter were showing another randomized letter. The letter-search task was designed to create a low to medium perceptual load.

Letter task Viewing task
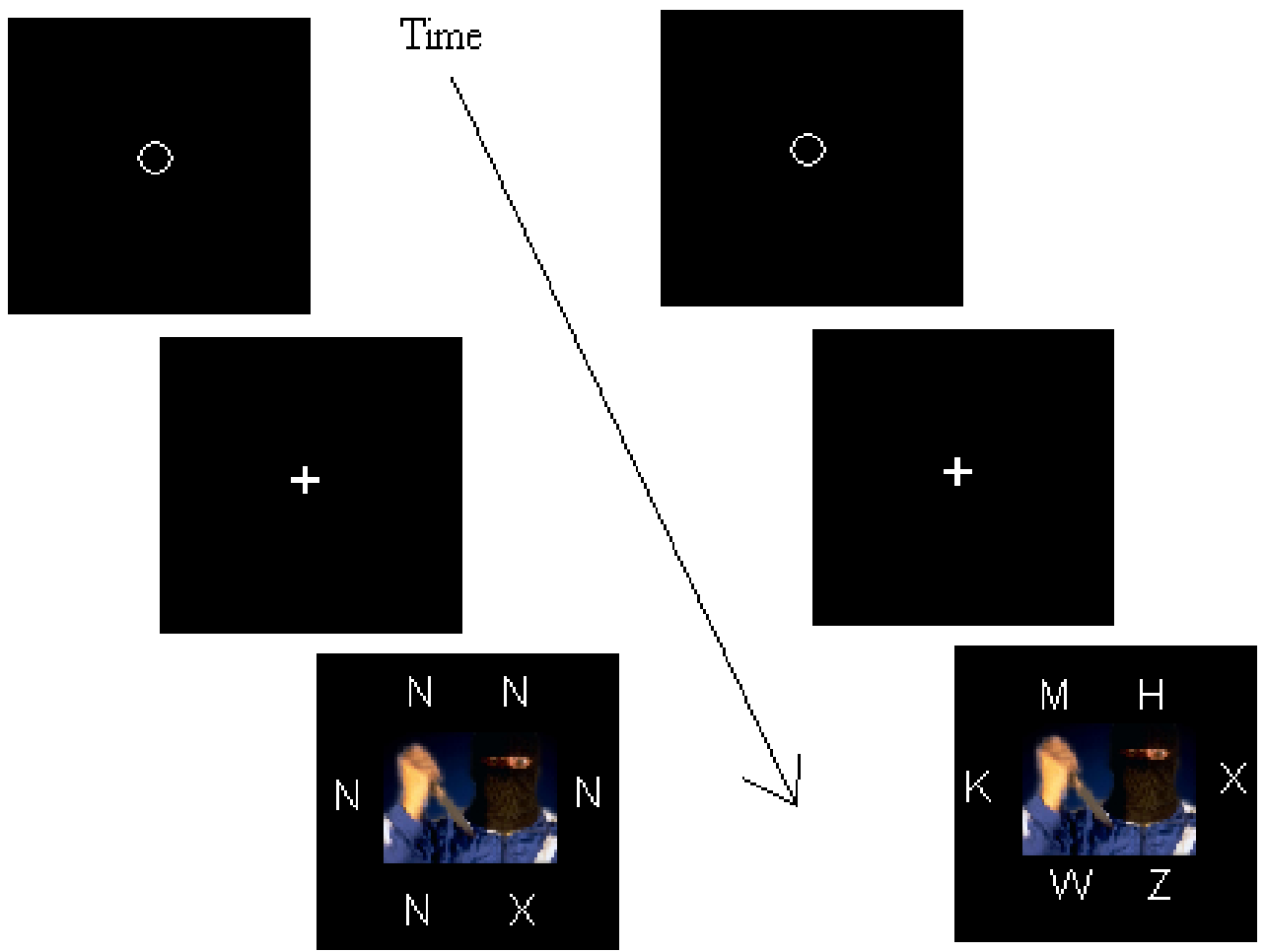

Figure 1. Experiment design, showing the time flow of what was presented onscreen. The black inter-stimulus screen, following each trial, is omitted from the presented succession.

Each trial began with a circle appearing for $1500 \mathrm{~ms}$, in the middle of the screen. This circle was followed by a fixation cross for a randomized period of $1000-1200 \mathrm{~ms}$, in the centre of the screen. The cross was followed by the 
stimuli presentation for $200 \mathrm{~ms}$. Figure 1 illustrates this design. Directly after the stimuli presentation, a black screen was shown for $1300 \mathrm{~ms}$. These four steps constituted a trial. At the end of a trial, the next one began automatically, until every trial for that condition was finished. As there were two different tasks, there were two recorded conditions, each consisting of 140 trials, preceded by two practice conditions of 10 trials each.

The stimuli presentation consisted of a rectangular IAPS image shown at centre screen, surrounded by six letters. The letters used were $\mathrm{M}, \mathrm{H}, \mathrm{W}, \mathrm{K}, \mathrm{Z}, \mathrm{X}$ and $\mathrm{N}$, (Lavie, 2005). The letter $\mathrm{N}$ appeared only as target, during the Letter task. Letters were randomized, in position and identity. Equally distanced from the image, letters were evenly spread, with two letters above the image, two letters below, and one flanking each side. This design was chosen as images were rectangular in shape and wider than they were tall.

Both tasks used the same stimuli, as randomized letters were always present during the viewing task, and randomized pictures were always shown during the letter task. The only difference between conditions, in form of stimuli, were the existence of the letter $\mathrm{N}$, only occurring during the letter task and the repetition of images, only occurring during the viewing task. This meant that the targets of one task could not be shown during the other task, and would not interfere with each other, eliminating the bias created by starting with the one or the other task.

Already at the stage of first contact, participants were informed about the necessary considerations, participant-wise, in EEG-testing, such as refraining from using hair-products like hairspray prior to the experiment. The method as such was also explained to the participant briefly, covering the usage of scalp electrodes and complimentary gel. Participants were also warned about the occurrence of offensive stimuli. At this point, none chose to end their commitment, although some opted for a rescheduling of appointments to times more suited for a bad hair day.

Participants were briefed on location about the exact details of the experiment. Details of the procedure was made clear, and participants signed an informed consent. Participants were instructed to refrain from movement in general, and eye blinking in particular. First, skull measurements were taken, and an EEGcap was tried and fitted for size. Then gel was applied to each electrode holder, and the electrodes themselves were plugged in.

Participants were seated in front of the computer screen, with their head supported by a chin rest. The distance between the eyes and the screen was 80 $\mathrm{cm}$. Order of the two tasks was counterbalanced across participants, so that in total, half began the experiment with the letter task, and the other half with the viewing task. The actual test-conditions were preceded by a respective trainingcondition for the particular task. The letter- and viewing-tasks were explained on screen at the beginning of each condition, and was first introduced and explained by the experimenter in a standardized fashion. For the viewing task, participants were instructed to complete the task by pressing the space-key every time a repetition of an image occurred, and ignore the letters presented. 
Similarly, for the letter task, participants were instructed to complete the task by pressing the space-key every time they saw an $\mathrm{N}$, and ignore the images presented. Participants were also instructed to blink only as the circle was shown on-screen, and keep their eyes at the fixation cross, at the centre of the screen. The instruction to keep eyes rested at the centre of the screen, at the fixation cross, remained the same over all conditions. The only differences in task instructions were in the difference of the tasks themselves, in the target to be searched for.

The same instructions and instructor were used for every experiment. Instructions were written and spoken in Swedish, however this differed somewhat on two occasions as two participants were foreign exchange students not familiar with the Swedish language, and so they were briefed and instructed in English instead. In an attempt to avoid expectancy effects, nothing was said concerning expected results or the hypothesis behind the experiment until the end of testing, at which time the rationale was explained and participants were offered a chance to stay informed of experiment results by means of email.

\section{Physiological Recording}

EEG activity was recorded at $512 \mathrm{~Hz}$ sampling rate (online low-pass filtered at $104 \mathrm{~Hz}$ ) from 128 active sites. The silver/silver-chloride $(\mathrm{AgAgCl})$ sinistered electrodes were mounted in an elastic cap and arranged in concentric rings with positions being at equiradial distance from the vertex. The EEG signal was digitized with BioSemi's system ActiveTwo, and was recorded with the built-in references (CML/DRS), using the dedicated software ActiView 604-Lores (www.biosemi.com). Data was only recorded during real trials, and never for practice trials.

\section{Data reduction}

As raw EEG data contains noise and artefacts generated by movement, it was pre-processed before analysis. For offline processing of recorded data BESA 5.0 (Brain Electrical Source Analysis) was used in this study. Data were referenced to linked ears. To reduce noise originating from electronic devices and wiring, a notch filter was applied to frequencies at $50 \mathrm{~Hz}$. Artefacts, in the form of eye blinks, were corrected for by adjusting models of typical blink patterns to individual participants, and identifying their blinks. The time period selected for analysis was $500 \mathrm{~ms}$ before image onset, to $1000 \mathrm{~ms}$ after onset, with $100 \mathrm{~ms}$ before onset set as baseline. Singular isolated noisy electrodes were interpolated. As movement artefacts are generated by pushing keys on the keyboard, all trials in which such pushing occurred were removed. Calculations for conditions were averaged between participants.

\section{Results}

\section{Behavioural Data}

Response time, target hits and misses and also false alarms were recorded and calculated. Figure 2 shows the relation between mean response times, neutral and negative stimuli, and task. Figure 3 illustrates the relation between mean 
number of hits, neutral and negative stimuli, and task. Behavioural data was subjected to repeated measures ANOVA's.

For response time (measured only for hits), the 2x2 repeated measures ANOVA of Task and Emotional content of image showed a significant main effect of task $F_{1,13}=5.768, p<.05\left(\eta_{2}=.307\right)^{1}$. There was no significant main effect of emotion $\left(F_{1,13}=1.169, p=.299\right)$ and no significant interaction effect of emotion $\mathrm{x}$ task $\left(F_{1,13}=0.020, p=.890\right)$. This $2 \times 2$ ANOVA is illustrated in figure 2 . The post hoc paired samples t-test of averaged response time between the task conditions showed it was during the letter task that response time was greater than during the viewing task $(p<.05)$. A paired comparison with task split by stimuli emotional content showed that there was a significant difference in response time when distracter shown was negative $(p=.02)$ and only a trend when the distracter was neutral $(p=.067)$.

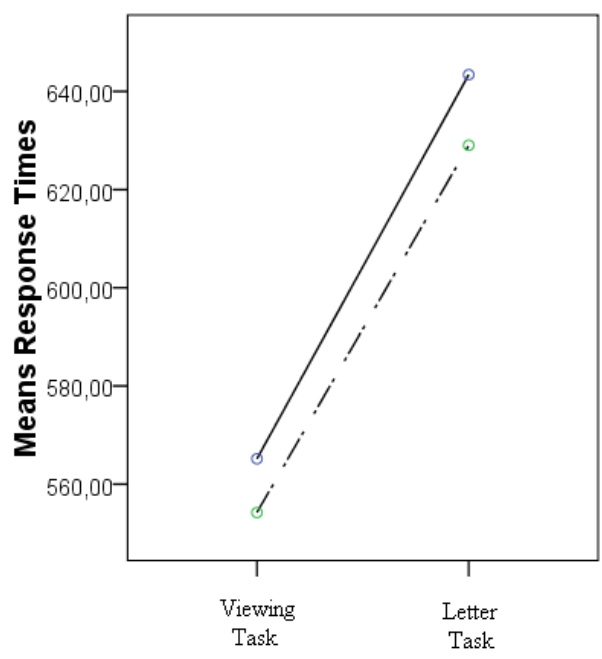

Figure 2. Response times, in milliseconds, for all conditions.

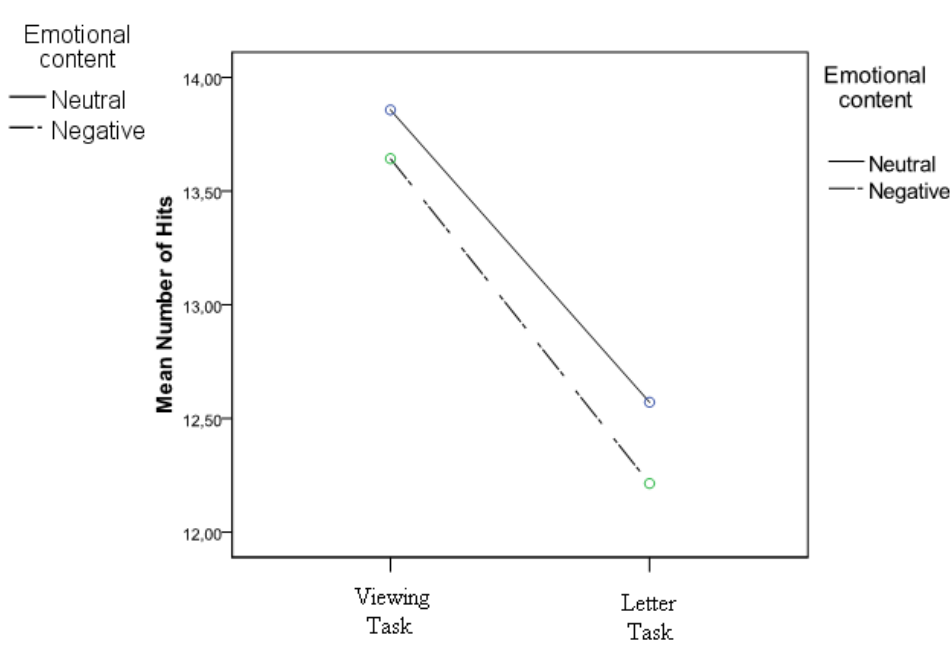

Figure 3. Mean number of hits, of 14 possible, for all conditions.

Target hits were counted as the times participants pressed the space key, when target stimuli was shown on-screen. A $2 \times 2$ repeated measures ANOVA for target hits showed a significant main effect of task $F_{1,13}=5.521, p<.05\left(\eta_{2}=\right.$ .298), but no significant main effect of emotional content in image $\left(F_{1,13}=1.434\right.$, $p=0.25)$ or interaction of task $\mathrm{x}$ emotion $\left(F_{1,13}=0.089, p=0.77\right)$. Figure 3 illustrates the above $2 \times 2$ ANOVA. A post hoc paired samples t-test of averaged target hits between the task conditions showed it was during the viewing task that the number of hits were greater than the letter task $(p<.05)$. Mean values of hits broken down per subconditions and averaged for particpants, with each subcondition consisting of 14 trials (14 possible hits): viewing task \& neutral stimuli: 13.86 hits $(S D=0.36) 99 \%$ correct, viewing task \& negative stimuli:

\footnotetext{
${ }^{1}$ A preliminary $2 \times 2 \times 2$ repeated measures ANOVA of Task, Emotional Content and Time within block, with Time within block as a two-level factor, split into a late and early group, showed no significant effect of Time within block $(p=0.618)$. Time within block was therefore not used in later analysis of data.
} 
13.64 hits $(S D=0.63) 97 \%$ correct, letter task \& neutral stimuli: $12.57(S D=$ 2.06) $90 \%$ correct, letter task \& negative stimuli: 12.21 ( $S D=2.30) 87 \%$ correct.

The times that participants pressed space, and the target stimuli was not present, were labelled false alarms. The $2 \times 2$ repeated measures ANOVA of false alarms showed no significant main effect of task, but a trend $\left(F_{1,13}=3.545\right.$, $p=0.082)$ for more false alarms during the letter task. There was no significant main effect of emotional content in image $\left(F_{1,13}=0.134, p=0.72\right)$ and no significant interaction effect of task $x$ emotion $\left(F_{1,13}=2,519, p=0.136\right)$. Mean values of false alarms broken down per subconditions and avareged for particpants, with each subcondition consisting of 46 trials (46 possible false alarms): viewing task \& neutral stimuli: 0.07 false alarms $(S D=0.27)$, viewing task \& negative stimuli: 0.21 false alarms $(S D=0.43)$, letter task \& neutral stimuli: 0.57 false alarms $(S D=0.94)$, letter task \& negative stimuli: 0.36 false alarms $(S D=0.63)$. In average, false alarms occurred in less than $1 \%$ of the 46 possible trials. Half of the participants (7) did not have any false alarms.

EEG analysis

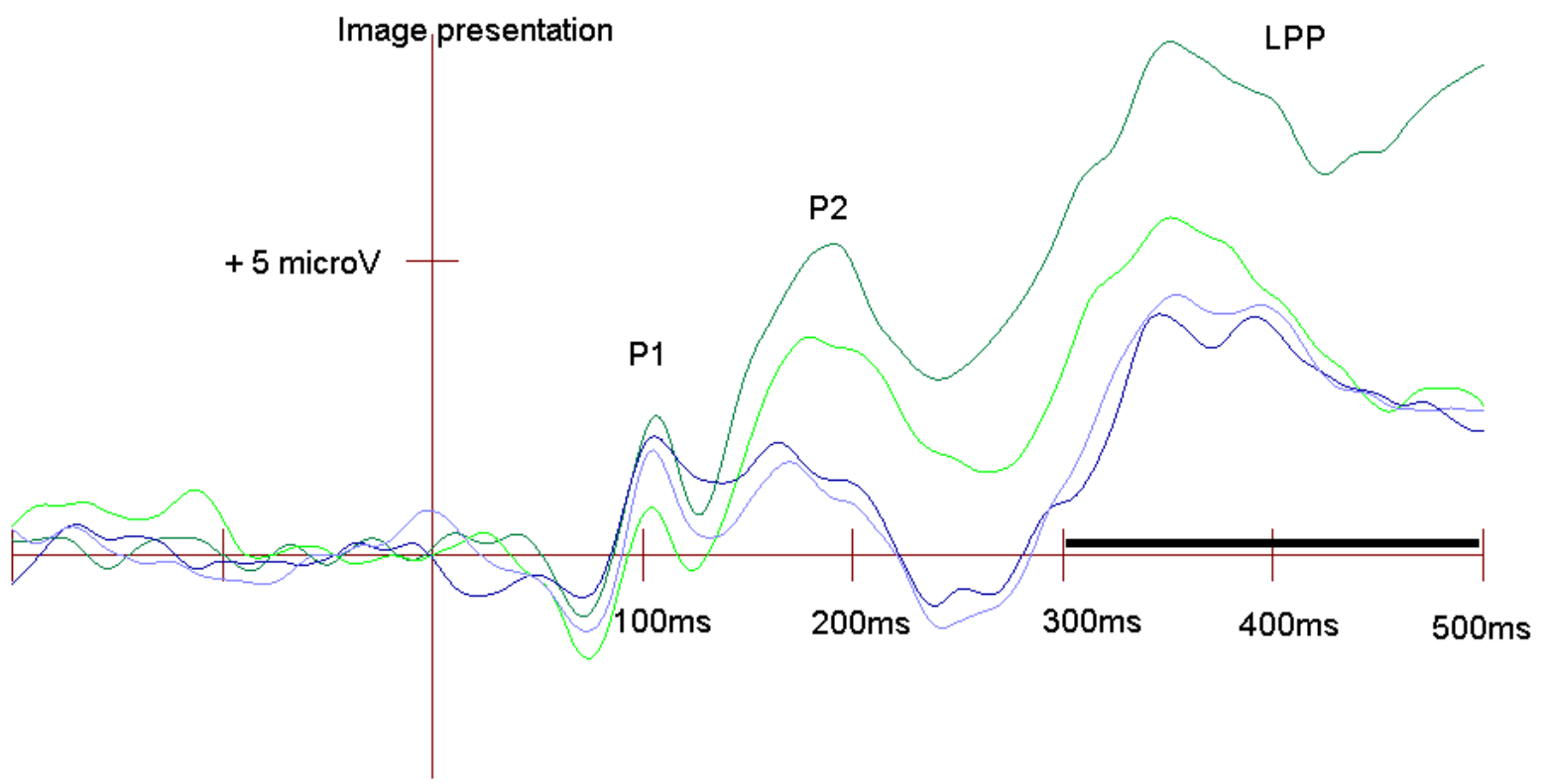

Viewing task and negative image

Letter task and negative image

Viewing task and neutral image

Letter task and neutral image

Figure 4. Individual ERP waves for four conditions (each assigned a colour) at electrode Pz. Reference was combined ears. The late positive potential (LPP) was computed as the mean amplitude at $\mathrm{Pz}$ between $300-500 \mathrm{~ms}$ (see black line).

Figure 4 shows the averaged waveforms over participants, at electrode $\mathrm{Pz}$, for the two tasks and the two different kinds of emotional images presented. Between 300-500 ms after picture onset, there was a strong positive amplitude for negative images showed during the viewing task (dark green waveform). 
While the waves of the three other conditions converge at some point after 400 $\mathrm{ms}$, this is not true for the negative images showed during the viewing task. The position of electrode $\mathrm{Pz}$ is shown in figure 5.

The 2x2 ANOVA for these conditions, showed a significant main effect of emotion $F_{1,13}=7.46, p$ $<.05$, with higher mean value for emotional images $^{2}$. There were near significant effects on LPP as averaged at Pz, of task $\left(F_{1,13}=3.57, p=\right.$ $0.081)$ and the interaction emotion $\mathrm{x}$ task $\left(F_{1,13}=\right.$ $3,23, p=0.095)$. Post hoc paired samples $\mathrm{t}-$ tests confirmed a significant difference in $\mathrm{Pz}$ activity for negative and neutral stimuli during the viewing task $(p=0.004)$ with greater activity

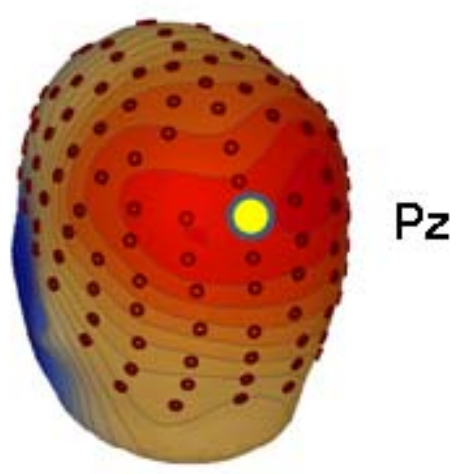

Figure 5. Position of electrode $\mathrm{Pz}$, marked by the dot, on the parietal lobe. at the negative stimuli and no significant difference between negative and emotional stimuli during letter task $(p=0.851)$. Post hoc paired samples t-tests also confirmed a significant difference between the tasks when both displayed emotional stimuli $(p=0.033)$, with larger activity during the viewing task, but there was no significant difference between tasks when both displayed neutral stimuli $(p=0.633)$.

Figure 6 illustrates the topography of the late positive potential (LPP) across the scalp, using the data collected from the experiment. At $500 \mathrm{~ms}$ following the presentation of unpleasant stimuli, there were distinctive positive amplitude changes, seen as two "peaks" at central-parietal, midline electrode sites during the viewing task. For the letter task, no clear difference in LPP activity can be seen in relation to emotional content of presented stimuli.

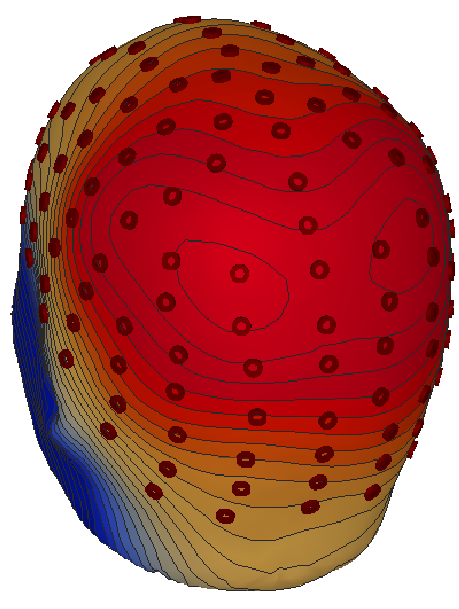

Viewing task
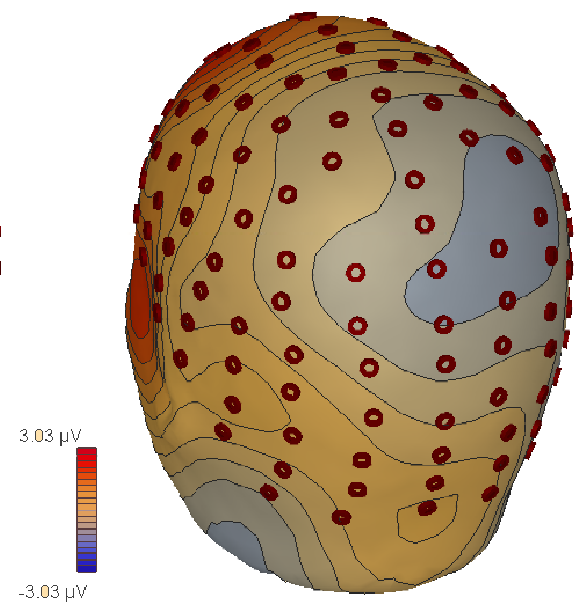

Letter task

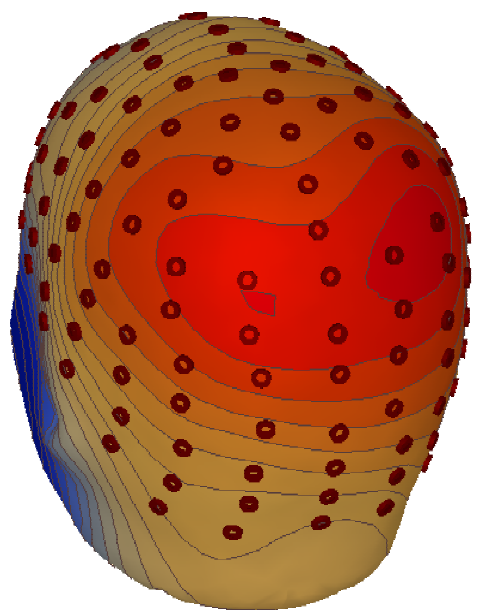

Differance

\footnotetext{
${ }^{2}$ A preliminary $2 \times 2$ repeated measures ANOVA of Task and Emotional Content using electrodes A03, A04, A05, A19, A32, A18, A20, and A31 (positioned around the Pz) also showed similar results, with a significant main effect of emotional content, $F_{1,13}=15,229, p<$ $.01(\eta 2=.539)$, with negative stimuli generating larger amplitudes.
} 
Figure 6. Topographies (head viewed from behind) for unpleasant minus neutral (i.e., difference waves) at $500 \mathrm{~ms}$ after picture onset during the viewing task (left), letter task (middle), and the task difference (viewing minus letter). The dots are electrode positions.

\section{Participant feedback}

Following the completion of each task, participants were asked some standard questions concerning task difficulty and other aspects concerning task completion. All participants responded that the letter task was harder, or more demanding, than the viewing task. Replies varied, but more than half (8 out of 14) rated the letter task as hard or very hard. When further inquired on the cause of this perceived difficulty, there was a clear trend to claim that the tasks were demanding in attention, especially so the letter task. Short exposure times and difficulties in seeing all of the letters at once (in the letter task) were cited as direct causes. Some participants pointed out a working-memory load as the factor of difficulty in the viewing task, though it was not necessarily referred to in such terms. In general, many participants expressed a difficulty in controlling eye blinks, as it was required of them to refrain from blinking, except for when the circle was shown on-screen. However, this requirement was stable across tasks.

\section{Discussion}

The experiment

Figures showed there to be a clear difference in measured amplitudes between conditions of neutral and emotional image content within the viewing task, but not the letter task. As hypothesised, clear positive potentials were observed at central-parietal, midline sites, during the 300-500 ms following visual stimuli, when participants were focused on image content, during the viewing task, and the images presented were emotionally negative as opposed to when they were neutral. This is interpreted as LPP activity, meaning that post-attentional processing of emotional stimuli was occurring, at least during the viewing task. Mean LPP amplitudes dropped for other conditions.

The 2x2 ANOVA of task and emotional content, using mean amplitudes at electrode $\mathrm{Pz}$ did not show significant effects (at the 0.05-level) of task or interaction of task and emotional content, but there were trends in the hypothesised direction. These trends, taken together with follow-up post hoc tests still indicate that LPP activity was greater during the viewing task than the letter task. The post hoc paired samples t-tests showed a significant difference between the tasks means when both displayed emotional stimuli, with larger activity during the viewing task, but there was no significant difference between tasks when both displayed neutral stimuli. Larger activity during the viewing task could be explained by it having a lower perceptual load. That there was no difference in activity when stimuli was neutral is coherent and predicted by the current understanding of LPP. 
Further, there was a significant difference between emotional and neutral images, in paired samples t-tests, during the viewing task, but not the letter task. This means that the emotional stimuli, when attended during the viewing task, was emotional enough to affect LPP measurements, but perhaps not significant enough for participants to override task focus during the letter task. The emotional stimuli were supposedly less attended in the letter task, as there was lower LPP amplitudes for those trials. Despite the lack of a main effect of task, or interaction of task and emotion in the 2x2 ANOVA, it is still clear that during the letter task, LPP activity showed no signs of discrimination between emotionally significant and insignificant stimuli. The reasoning behind using paired samples t-tests was to investigate the existence of LPP activity during the two tasks, when viewed separately. LPP activity, in this study, could not be measured as a stand-alone positivity, but rather as a positivity following emotional images in relation to the neutral images. As LPP signatures emotional processing, and emotional processing would not take place for emotionally neutral stimuli, there would be a significant difference between the two conditions (emotional and neutral images), if emotional processing was occurring during any task. Also, in EEG, reference points may be affected by surrounding activity, that is related to the specific task, yet not related to emotions or LPP. This can become a problem, when using raw numbers to estimate effects over different tasks. Taken together, these reasons were thought to justify paired samples t-tests. The lack of significant differences in LPP activity for negative and neutral emotional pictures shown during the letter task, in the paired samples t-test, should probably be interpreted as there being no meaningful LPP activity during the letter task. This could indicate that conscious processes of task relevance were prioritised as opposed to unconscious automatic emotional processes, that would draw attention to emotionally significant stimuli instead of task-significant stimuli. It could mean that the pictures were successfully ignored, partially or completely, when attention was focused on the letter task.

The predictions that LPP activity would be lower for the more perceptually demanding of the two tasks, and also lower for neutral as opposed to emotional images presented seems confirmed. Participant feedback, response time and number of hits all pointed towards the same conclusion, that the letter task was in fact harder than the viewing task. This was as intended, as a task including a perceptual load should be harder than one that does not. Further, participant feedback placed this difficulty on a perceptual level, consistent with the goal of creating a perceptual load. Diminished LPP effects of negative stimuli presented during the letter task is also consistent with this. Taken as a whole, all of the results are consistent with Load Theory, given that the letter task did indeed involve a sufficiently demanding perceptual load.

Even though the letter task at the drawing board stage was made out to be a task of low to medium perceptual load, it quickly became apparent, through participant feedback, that it was in fact a higher load than intended. While this makes it useless to increase the level of perceptual load of the task, it might still be possible to lower it, by reducing the number of potential targets in the letter task or perhaps increasing exposure time. As the purpose of this study was only to set the stage for following experiments, with varied conditions of load, there 
was no other measurement of difficulty or load, except what could be drawn from feedback and existing data. It was however clear that the level of difficulty, as measured in hits or false alarms, was low, even in the letter task, compared to other studies of load. That participants generally did very well, yet reported that the letter task was very demanding, could indicate that perceptual load was probably distinguishable from task difficulty, even though participants did report that the more perceptually demanding task was also the more difficult. Participants did explicitly place the difficulty of the task on a perceptual level, and the stimuli shown was the same for all conditions. Though the nature of the letter task and the viewing task are quite different.

It could be argued that the one-back task (viewing task), being a task in which one has to keep in memory the information from the previous image, creates a working-memory load. As shown by Lavie (2005) a high-working memory load leads to increased distracter interference. But a low working memory load has the opposite effect, by decreasing distracter interference. It is unclear at what exact level the working memory load was, but even though objective ratings were not used, there was no trend in participant feedback to rate the viewing task as demanding. Though participants did report that there was some level of working-memory load, and that this added to the difficulty. Also, measures of LPP should not be affected by concurrent tasks (Hajcak et al., 2007). The rationale behind using a one-back task was to get participants to actively view the images, in a straight-forward task, and allow for quick responses related to directly observable image properties.

That the letter task and the viewing task focused on different aspects of the stimuli presented should not matter for early processing, as the fixation cross remained at the middle of the screen, and participants were instructed to keep their eyes rested at that point. Covert attention seemed to function then, and participants were able to distinguish clearly between targets and non-targets, even during the letter task. The fact that letters were not spread evenly, as pictures were wider than tall, did not seem to cause eye-movements, and as eyes comes in pairs of two, the human visual field is also wider than it is tall.

The main difference from other Load Theory experiments, was that this used distracters that were not potential targets of the task. Even though distracters and targets appeared on the same screen and visual field, during the letter-task, they were clearly separated not only by their positions, but also in other physical features. The similarity of stimuli is definitely a factor, as it is more difficult to ignore distracters that are similar to the targets in physical properties such as shape and colour (Koivisto \& Revonsuo, 2008). The distracters used in this experiment were very dissimilar to the targets, during the letter-task, as the letters were always in the same tone of gray, the same case, and composition. Images instead featured a varied palette of colours, different motives, and composition. This makes early selection easy, and distracters during the letter task can thus be filtered without any need for later processing like object recognition. Though the focus of this study was different than earlier Load Theory experiments, in exploring the effects of emotionally significant, but taskirrelevant stimuli under load conditions, and using other measurements, so this might not be an issue. Emotionally significant stimuli should draw attention 
automatically, as long as perceptual resources still remain. Also, the dimension of compatible versus incompatible distracters (often used in studies of perceptual load) were here replaced with the dimension of emotionally significant versus insignificant.

This study did not include any other awareness-check in relation to stimulus than measured LPP. It could be that subjects were still aware of the irrelevant stimuli to some degree when it was emotionally significant, despite low LPP activity. This makes it hard to draw conclusions about the occurrence of early selection. Generally, it is important to note that even though there may be no significant levels of activity, it does not follow that there is no activity at all. It might just be that only a weak signal is generated, that is existent, but not statistically significant or otherwise easy to perceive.

Although similar in design to the experiment on spider-fearful individuals, where the spider-fearful individuals still showed measurable LPP differences to emotional and neutral stimuli during a high-load task (Norberg et al., 2009), there were some differences in method. First, this experiment used IAPS images, that were more complex and the images were in colour for this experiment, but in black and white for the spider-study. Second, the instructions differed, and the presentation of a circle as a blinking cue was new to this experiment. Third, the size and shape of the pictures and letters differed. And last, but most importantly, the participants in the spider-study were selected on the basis of being either very fearful, or non-fearful of spiders, creating two extreme groups with different relations to the stimuli presented.

Image size can affect measures of LPP, and smaller images generally produce smaller positivity's (de Cesarei \& Codispoti, 2006). This would not explain why emotionally significant stimuli was more easily ignored in this study, compared to the spider-study, as this study used larger images. An effect of image size is however the positions of the letters surrounding the pictures. With a larger image, the letters will be further apart and at a longer distance from the centre, and letters are harder to perceive in the periphery of eye. Supposedly, this creates a greater difficulty. This difficulty might be unaffected by perceptual resources, as the clearness of the letters could be considered a form of data limits, and data limits should not affect distracter interference (Lavie, 2005). However, as Lavie (2005) refer to clearness in the sense of manipulating images, and not their position on a retinal field, it could remain unclear whether placing objects in the periphery effects distracter interference. Now then, it is time to move on from the issue of larger pictures, to the larger picture.

\section{Theories and Future}

The hypothesis of Load Theory was that LPP amplitudes would be larger during the viewing task and lower during the letter task, as an effect of perceptual load. As this was the case, the study can be seen to support Load Theory. LPP results from this study cannot, however, say much about if selection occurred early or late, as there was only one condition of load, and load was not varied in similar tasks. 
As mentioned earlier, in the similar experiment conducted on spider-fearful and non-fearful participants, a high perceptual load did not eliminate LPP to pictures of spiders, when presented to spider-fearful participants, as task-irrelevant stimuli (Norberg et al., 2009). As this experiment was similar to that of spiderfearful individuals, but yielded different results, several possible conclusions can arise. It should not be the case that that the emotionally arousing pictures in this current study were not emotional enough, as standardised arousal ratings place them in the highest category. Though their higher level of complexity could make them slower to process. Another possibility is that some evolutionary threats, such as spiders, trigger facilitated attention, while other negative emotional stimuli do not, simply because facilitated attention towards them has not aided evolutionary progress. While spiders can be avoided, if noticed, other negative emotional stimuli cannot. Further, some negative emotional stimuli, such as mutilated bodies, might elicit disgust responses, but not pose an immediate danger.

It might be that results from the study of spider-fearful individuals provide an exception to Load Theory. Load Theory indicates that conscious task-focus will always be prioritised over automatic responses, given a high enough load. This might not be the case, as the kind of extremely emotionally significant stimuli that spiders represent to spider-fearful individuals might be prioritised over all else. A conscious system need not necessarily override an unconscious one. From an evolutionary perspective, it would seem plausible that automatic systems sometimes override conscious ones, as poisonous spiders are lifethreatening, and the struggle for life and offspring the driving force of evolution (Buss, 2009). As the experiments were different, even though they shared similarities, it might not be reasonable to generalise completely between them. It would be preferable to use the exact same design and conditions in future studies, exploring different types of emotional stimuli. Apart from spiders and snakes, there might be other forms of stimuli that receive the same prioritised processing. Imminent dangers such as other predator threats or rapidly approaching objects could produce the same results.

For theories of selection and early and late processing, it could be useful to consider two dimensions in affecting selection. First, there may be consciously affected selection, in the form of task relevance and guided attention to some certain stimuli, in a larger set of stimuli. Consciousness should here be understood as the process of having explicit knowledge of and access to the selection process. In practice, conscious processes can be the subject of explicit recall and can be monitored by awareness. We might, for instance, seek to buy a certain breakfast cereal from a store. As not any cereal will do, we have consciously decided upon, after careful deliberation, which one to seek out. All other cereals and products that we see in this store are rejected, deemed as irrelevant stimuli. This selection affection is voluntary. Then there is unconscious selection affection, that is automatic and usually occurs at an early stage of perception. We might base assumptions and answers on these processes, but we cannot directly access them. Situations occur, for example, where participants seem to perform perception tasks above chance level, yet remain unaware of the process and information they use to guide their performance (Wiens \& Öhman, 2002). As proposed earlier, emotionally 
significant stimuli, especially life-threatening, will draw to it our attention. Should a sabre-toothed tiger suddenly leap out from behind a store shelf, we just might find ourselves looking at it, and also acting automatically upon that stimuli, perhaps mindlessly fleeing, regardless of the importance of our current search for our favourite cereal. However, had this tiger not been emotionally significant to us, its presence might not have entered consciousness, or affected action. The sort of scanning process that created a response to the tiger, and made it significant, may not be directly accessible to us, but automatic. Some unconscious processes that affect selection might be hard to influence, as in the case of phobias, while conscious processes are more adaptable.

In the widest of theoretical application, these sorts of experiments might be able to tell us something about the interaction of conscious and unconscious systems, as part of a wider field. Unconscious determinants affect decision making and attention. To understand how these operate, one piece of the puzzle is to understand how and when stimuli irrelevant to conscious operations still receive processing resources. Stimuli can be filtered as irrelevant, by conscious systems, yet relevant by unconscious ones, resulting in competition. Being that the winner of this competition is the one actually affecting our behaviour, while the loser might not, this is a fundamental question. As stimuli are always competing with other stimuli, it becomes essential to understand how the process of selection works, as this process is the base for later processing systems of memory, motor reactions, planned voluntary actions and ultimately directly effects how we come to act in our world. How, and when, a viewer reacts to stimuli that are consciously rated as irrelevant, can in effect show how relevant that conscious rating of relevance is to actual behaviour. It could be, that the conscious process of placing task relevance, is sometimes, in itself, irrelevant.

\section{References}

Bahrami, B., Carmel, D., Walsh, V., Rees, G., \& Lavie, N. (2008). Unconscious orientation processing depends on perceptual load. Journal of Vision, 12, 1-10.

Beck, D. M., \& Lavie, N. (2005). Look Here but Ignore What You See: Effects of Distractors at Fixation. Journal of Experimental Psychology: Human Perception and Performance, 31, 592-607

Buss, D. M. (2009). The great struggles of life: Darwin and the emergence of evolutionary psychology. Am Psychol, 64, 140-8.

Conway, A. R. A., Cowan, N., Bunting M. F. (2001). The cocktail party phenomenon revisited: The importance of working memory capacity. Psychonomic Bulleting and Review, 8, 331-335.

De Cesarei, A., \& Codispoti, M. (2006). When does size not matter? Effects of stimulus size on affective modulation. Psychophysiology, 43, 207-215.

Devue, C., Laloyaux, C., Feyers, D., Theeuwes, J., \& Brédart, S. (2009). Do pictures of faces, and which ones, capture attention in the inattentional-blindness paradigm? Perception 38, 552 - 568

Hajcak, G., Dunning, J. P., \& Foti, D. (2007) Neural Response to Emotional Pictures Is Unaffected by Concurrent Task Difficulty: An Event-Related Potential Study. Journal of Experimental Psychology: Human Perception and Performance, 34, 1078-1091. 
Koivisto, M., \& Revonsuo, A. (2008). The role of unattended distractors in sustained inattentional blindness. Psychological Research, 72, 39-48

Lachter, J., Forster, K. I., Ruthruff, E. (2004). Forty-five years after Broadbent (1958): Still no identification without detection. Psychological Review, 111, 880-913.

Lang, P. J., Bradley, M. M., \& Cuthbert, B. N. (2008). International affective picture system (IAPS): Affective ratings of pictures and instruction manual. Technical Report A-8. University of Florida, Gainesville, FL.

Lavie, N. (1995). Perceptual Load as a Necessary Condition for Selective Attention. Journal of Experimental Psychology: Human Perceptions and Performance, 21, 451-468.

Lavie, N. (2005). Distracted and confused?: Selective attention under load. TRENDS in Cognitive Sciences, 9, 75-82.

Lavie, N., \& Fockert, J. W. (2003). Contrasting effects of sensory limits and capacity limits in visual selective attention. Perception \& psychophysics, 65, 202-212.

Lavie, N., \& Tsal, Y. (1994). Perceptual load as a major determinant of the locus of selection in visual attention. Perception \& Psychophysics, 56, 183-197.

Luck, S. J. (2005). An Introduction to the Event-Related Potential Technique. Cambridge, Mass: The MIT Press.

Macdonald, J. S. P., \& Lavie, N. (2008). Load Induced Blindness. Journal of Experimental Psychology: Human Perception and Performance, 34, 1078-1091.

Norberg, J., Wiens, S., \& Peira, N. (2009). Never mind the spider: Late positive potentials to phobic threat at fixation are unaffected by perceptual load. (in press)

Pastor, M. C., Bradley, M. M., Löw, A., Versace, F., Moltó, J., \& Lang, P. J. (2008). Affective picture perception: Emotion, context, and the late positive potential. Brain Research, 1189, 145-151.

Rakison, D. H., \& Derringer, J. (2008). Do infants possess an evolved spider-detection mechanism? Cognition, 107, 381.

Rees, G., Frith, C. D., \& Lavie, N. (1997). Modulating irrelevant motion perception by varying attentional load in an unrelated task. Science, 278, 1616-9.

Schupp, H. T., Cuthbert, B. N., Bradley, M. M., Cacioppo, J. T., Ito, T., \& Lang, P. J. (2000). Affective picture processing: The late positive potential is modulated by motivational relevance. Psychophysiology $37,257-261$.

White, R. C., \& Davies, A. A. (2008). Attention Set for Number: Expectation and Perceptual Load in Inattentional Blindness. Journal of Experimental Psychology: Human Perception and Performance, 34, $1092-1107$.

Wiens, S., \& Öhman, A. (2002). Unawareness is more than a chance event: comment on Lovibond and Shanks. Journal of Experimental Psychology: Animal Behaviour Processes, 28, 27-31.

Öhman, A., Flykt, A., \& Esteves, F. (2001). Emotion drives attention: Detecting the snake in the grass. Journal of Experimental Psychology: General, 130, 466-478. 